\title{
CORRECTIONS
}

\section{Paul Harald Osterberg}

Due to an editorial error, this Obituary for Paul Harald Osterberg has been attributed to the wrong author (BMJ 2013;347:f4851, doi:10.1136/bmj.f4851). The author is Vanessa Larmor, not Pier Milner. 